\title{
CORRIGENDUM
}

\section{Towards a Holistic Environmental Flow Regime in Chile: Providing for Ecosystem Health and Indigenous Rights - CORRIGENDUM}

Elizabeth J. Macpherson and Pia Weber Salazar

https://doi:10.1017/S2047102520000254, published by Cambridge University Press, 2 October 2020.

Keywords: Environmental flows, Cultural flows, Indigenous water rights, Water equity and distribution, Chile, Corrigendum

When originally published, the article by Elizabeth Macpherson and Pia Weber Salazar included an incorrect footnote. Footnote 258 on page 39 should have read:

Marín, n. 15 above, p. 110.

\section{REFERENCE}

E.J. Macpherson and P. Weber Salazar, 'Towards a Holistic Environmental Flow Regime in Chile: Providing for Ecosystem Health and Indigenous Rights' (2020) 9(3) Transnational Environmental Law, pp. 481-519. 